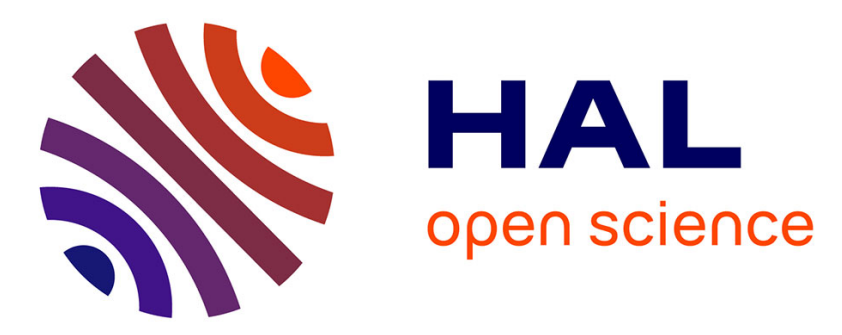

\title{
A joint reflectometry-optimization algorithm for mapping the topology of an unknown wire network
}

\author{
Moussa Kafal, Jaume Benoit, Christophe Layer
}

\section{To cite this version:}

Moussa Kafal, Jaume Benoit, Christophe Layer. A joint reflectometry-optimization algorithm for mapping the topology of an unknown wire network. 2017 IEEE SENSORS, Oct 2017, Glasgow, United Kingdom. pp.8234208, 10.1109/ICSENS.2017.8234208 . cea-01817859

HAL Id: cea-01817859

https://hal-cea.archives-ouvertes.fr/cea-01817859

Submitted on 27 Jan 2021

HAL is a multi-disciplinary open access archive for the deposit and dissemination of scientific research documents, whether they are published or not. The documents may come from teaching and research institutions in France or abroad, or from public or private research centers.
L'archive ouverte pluridisciplinaire HAL, est destinée au dépôt et à la diffusion de documents scientifiques de niveau recherche, publiés ou non, émanant des établissements d'enseignement et de recherche français ou étrangers, des laboratoires publics ou privés. 
See discussions, stats, and author profiles for this publication at: https://www.researchgate.net/publication/319058894

\section{A Joint Reflectometry-Optimization Algorithm for Mapping the Topology of an Unknown Wire Network}

Conference Paper · October 2017

DOI: 10.1109/ICSENS.2017.8234208

CITATIONS

9

3 authors, including:

Moussa Kafal

Atomic Energy and Alternative Energies Commission

40 PUBLICATIONS 191 CITATIONS

SEE PROFILE

Some of the authors of this publication are also working on these related projects:

Fault Detection and Location of Power Networks View project

Project Imaging Techniques for soft fault detection and location in wiring networks View project
READS

166

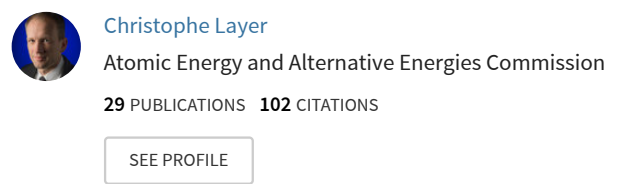




\title{
A Joint Reflectometry-Optimization Algorithm for Mapping the Topology of an Unknown Wire Network
}

\author{
Moussa Kafal, Jaume Benoit, Christophe Layer \\ CEA, LIST, Laboratoire de Fiabilisation et d'Intégration des Capteurs \\ Nano-Innov, Bât. 862-PC172, 91191 Gif-sur-Yvette Cedex, France \\ \{name.surname\}@cea.fr
}

\begin{abstract}
Despite the fact that wireless systems are overwhelming most of nowadays applications, wiring networks are still forming a pivotal anchor in many controls and security units. Thus, ensuring the reliable operation of such networks necessitates investing in techniques dedicated for their protection. A vast majority of such methods rely on the presence of a reference model of the network. In this paper, we propose a nondestructive testing approach based on the tenets of reflectometry methods and genetic algorithms to retrieve the topology and load impedances of unknown embedded complex wiring networks.
\end{abstract}

Keywords-Wiring networks, topology reconstruction, time domain reflectometry, genetic algorithms.

\section{INTRODUCTION}

Although electrical wires are often the last component taken into account during system designs, it won't be exaggerating to consider them as the body's nervous system. In fact, wires not only distribute power but also provide control and information links between multiple systems of many applications. Some are critically dangerous as nuclear power plants and aircraft industry. On the other hand, wires are subject to aging during their normal service life leading to the progressive deterioration of their physical properties and performance. Consequently, the potential hazard posed by wiring faults has generated a tremendous amount of effort invested in developing robust techniques for their detection as an early warning approach for ensuring a trustworthy usage of cables.

Although reflectometry-based techniques formed a pivotal anchor towards this target [1], major concerns sieged their feasibility due to several reasons. Essentially based on analyzing the reflected echoes generated by injecting a test signal into the network under test (NUT), weak echoes produced by soft faults, which are minor alterations that affect a cable, can pass undetected with respect to spurious signals propagating over an NUT. Such signals can be mechanical vibrations or useful communication signals as in live wire testing. A further challenging level of complexity is added to the reflectometry response when dealing with networks interconnecting several branches via junctions which is often the case in almost all real-life environments [2]. In fact, junctions create echoes that can either mask those generated by soft faults or be difficult to distinguish from those reflected by hard faults (open or short circuits). Regrettably, such undesired echoes tend to grow exponentially with an increased complexity of the NUT [3].

Within this context, baselining, a method based on comparing the faulty NUT's response with a predefined healthy one, enhanced the reflectometry's sensitivity towards detecting weak or masked faults' signatures [4]. Besides, it enabled applying a new category of time-reversal based methods that have shown promising results in locating multiple soft faults in different complexity NUTs [5]-[7]. Consequently, analyzing networks of unknown topology can therefore be particularly very difficult. This is supported by the wide range of networks whose configuration datasheets are lost especially when dealing with old wiring infrastructures. Furthermore, disconnecting embedded NUTs for testing purposes in some applications can sometimes be impossible which in turn imposes two serious constraints demonstrated first by the limited number of available testing ports that can be a single one in many cases. Second, we might be confronted by load impedances that could not be disconnected and changed.

Thus, the aim of this paper is to propose an efficient automated method to retrieve all parameters of an unknown or partially unknown wiring network, namely its topology and extremities' load impedances. A forward model is developed to generate the time domain reflectometry responses (TDR) of NUTs and the genetic algorithm (GA) is used to solve the inverse problem in order to reconstruct the right network topology. This new TDR-GA algorithm will be accomplished using a single-port access measurement without any need to disconnect the tested network.

\section{THE JOINT TDR-GA APPROACH}

The joint TDR-GA approach consists of two main parts. The first part comprises a numerical model for constructing NUTs in addition to a transmission line forward modeling to generate their corresponding TDR responses. The second part is the GA optimization which is used to minimize the differences between the measured reflectometry response of an unknown NUT and the responses given by the direct numerical model. The network's topology and its corresponding load impedances would be retrieved once convergence is reached between the measured response and the one corresponding to the right NUT configuration generated by the forward model. It is worthy to note that we will be dealing with NUTs composed of cables having the same characteristic impedance $Z_{c}$.

\section{A. Numerical model}

Based on the fact that the number of extremities of a black-boxed unknown network can be known, a numerical model is designed to construct all possible NUTs connecting these extremities. During this procedure all ring and mesh like configurations are dropped, where only tree like (branched) 
topologies will be maintained. In fact, this assumption was based on the event that branched networks are more often found in practice than other NUT topologies [8].

In order to model the signal propagation in a branched NUT and provide its corresponding TDR response, a joint finite difference time domain (FDTD)- ABCD matrix method developed as an inhouse solver at CEA was adopted. It is a simple electromagnetic modeling tool which provides very computationally efficient time domain voltage $(v)$ and current (i) solutions to Telegraph's equations given by (1) and (2) of the RLCG circuit model used to describe the wave propagation in a two-conductor transmission line. The quantities $R$ (resistance), $L$ (inductance), $C$ (capacitance) and $G$ (conductance) are the per unit length parameters.

$$
\begin{gathered}
\frac{\partial v(x, t)}{\partial x}=-R i(x, t)-L \frac{\partial i(x, t)}{\partial t} \\
\frac{\partial i(x, t)}{\partial x}=-G v(x, t)-C \frac{\partial v(x, t)}{\partial t}
\end{gathered}
$$

\section{B. The Genetic Algorithm}

Genetic Algorithms (GAs) are adaptive heuristic search algorithms based on the evolutionary ideas of natural selection and genetics [9]. As such, they represent an intelligent exploitation of a random search used to solve optimization problems.

GAs simulate the survival of the fittest by starting with a random sample set (initial population) of potential solutions (chromosomes). Each chromosome is coded as a finite length vector of variables which are analogous to genes. These solutions then undergo recombination and mutation (like in natural genetics), producing new children, and the process is repeated over various generations. Each individual (or candidate solution) is assigned a fitness value (based on its objective function value) and the fitter individuals are given a higher chance to mate and yield more "fitter" individuals until an eventual convergence is reached. As a result, an optimal population is produced that will now contain the optimal solutions.

\section{The TDR-GA Approach}

After measuring the reflectometry response $V_{\text {mes }}$ of the unknown black-boxed network $N U T_{m e s}$ using a single testing port, the first part of the TDR-GA approach begins by numerically constructing all $N$ possible NUT configurations $N U T_{\text {sim }}^{i}$ $(i=1,2, \ldots, N)$ based on the knowledge of the $N U T_{m e s}$ 's $m$ extremities. The TDR response $V_{\text {sim }}^{i}$ of each $N U T_{\text {sim }}^{i}$ is determined by the numerical model described section II-A.

Since the GA algorithm has shown to be a feasible and efficient tool for solving difficult optimization problems, it will be used to minimize the differences between $V_{m e s}$ and each $V_{\text {sim }}^{i}$ in an effort to retrieve the best $N U T_{\text {sim }}^{i}$ that best resembles $N U T_{m e s}$. Accordingly, we will start with an initial population which is a random selection of possible solutions. Each chromosome is a model vector $X=[L, Z]$, with $L=\left[L_{1}, L_{2}, \ldots, L_{n}\right]$ being the lengths of $n$ branches composing the $N U T_{\text {sim }}^{i}$, while $Z=\left[Z_{1}, Z_{2}, \ldots, Z_{m}\right]$ being the load impedances of its $m$ extremities. These solutions then undergo the GA process described in sec. II-B with an objective function value defined as $F_{v a l}^{i}=\left\|V_{s i m}^{i}-V_{m e s}\right\|_{2}$. After each GA process the fitness values $F_{v a l}^{i}$ will be sorted and the $N U T_{s i m}^{i}$ with the highest $F_{v a l}^{i}$ will be dropped. This process will be iterated till we reach a stopping criterion demonstrated by the lowest $F_{v a l}^{i}$ corresponding to $N U T_{\text {sim }}^{i}$ whose solution $X$ best resembles the $N U T_{m e s}$. For clarification purposes, the diagram of Fig. 1 summarizes all the steps we followed in the proposed TDR-GA algorithm.

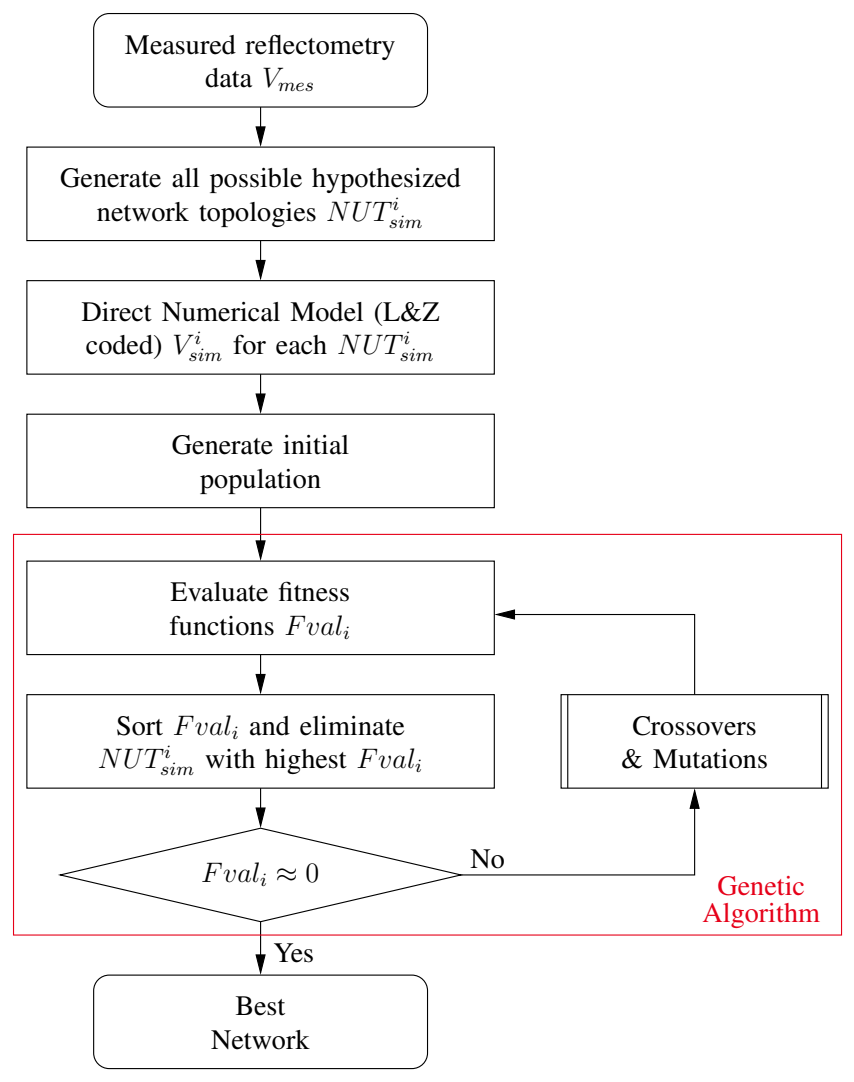

Fig. 1. The TDR-GA algorithm.

\section{RESULTS AND DISCUSSIONS}

In order to validate the proposed approach, we opted to test experimentally a double Y-junction NUT composed of $50 \Omega$ coaxial cables connected to different resistive loads as shown in Fig. 2. The lengths of branches as well as load impedances are illustrated on the same figure.

The standard TDR measurement was accomplished by injecting a Gaussian pulse excitation with a $-3 \mathrm{~dB}$ bandwidth

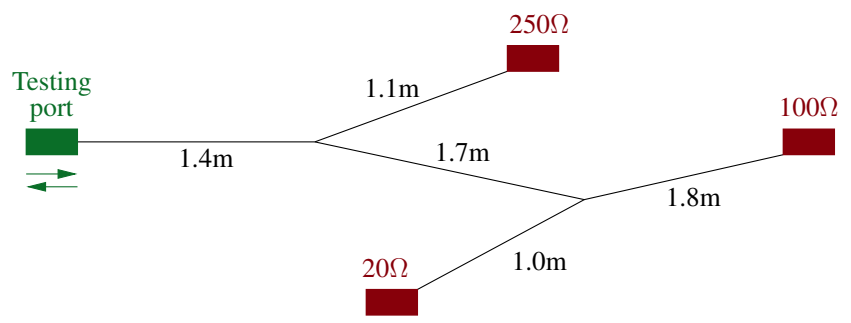

Fig. 2. Layout of the double Y-junction NUT considered for the experimental validation of the TDR-GA approach showing the branch lengths and the load impedances. 


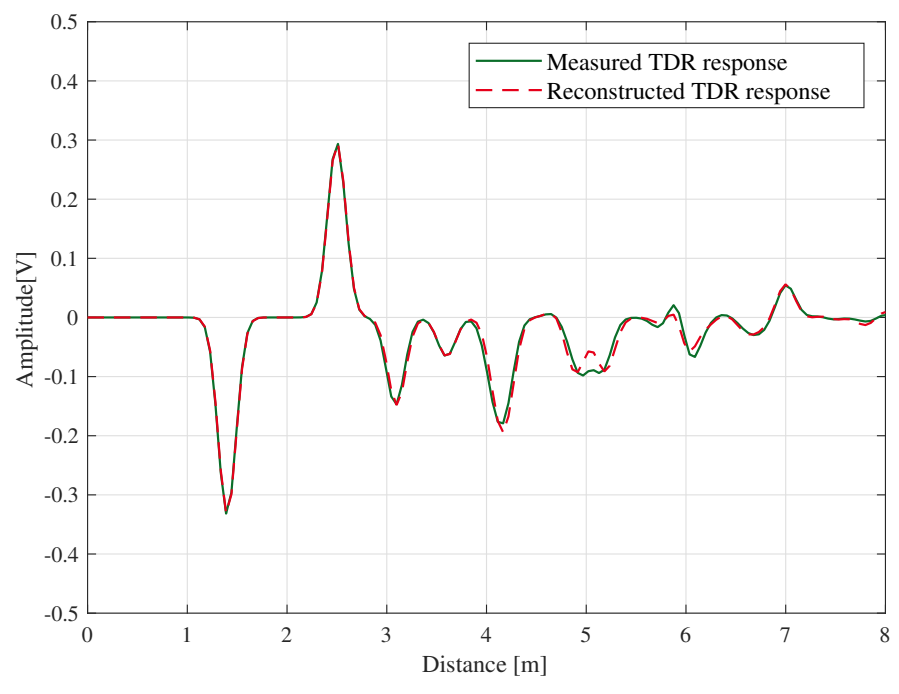

Fig. 3. The measured and simulation-reconstructed reflectometry responses of the unknown network.
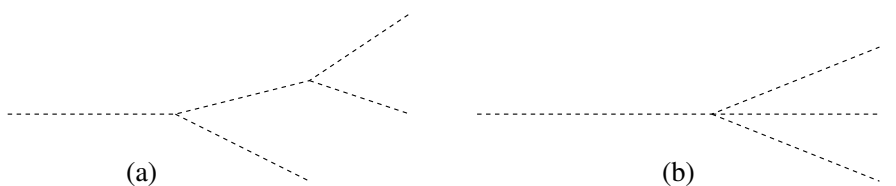

Fig. 4. The hypothesized numerical NUTs constructed based on the 4 extremities of the tested NUT: (a) a double Y-junction NUT, (b) a single Y-junction NUT.

of about $2 \mathrm{GHz}$ into the testing port of the network. The TDR response is retrieved by measuring the $S_{11}$ parameter of the NUT by a vector network analyzer Rohde \& Schwarz ZVB8 VNA, covering a frequency range from $300 \mathrm{kHz}$ to $8 \mathrm{GHz}$. The reflectogram of Fig. 3 shows the measured time domain TDR response of the NUT after applying an inverse fast Fourier transform (IFFT) to the frequency domain measured response.

The very first step of the TDR-GA algorithm starts by numerically constructing all possible hypothesized network topologies based on the diagnosed NUT's number of extremities. Thus, based on the knowledge of 4 extremities, we can build two different tree like NUTs as shown in Fig. 4. This will be followed by applying the procedure detailed in sec. II-B which seems to exclude the hypothesized NUT of Fig. 4 (b) after the first iteration and keeps us with only the NUT of Fig. 4 (a) qualified for the second round. Consequently, the second iteration of the approach optimizes the solutions (branch lengths and load impedances) of the NUT leading to the network of Fig. 5 in 182 seconds using a standard stateof-the art computer. The comparison between the obtained NUT with the original measured network shows that the TDRGA approach efficiently determined the topology of the tested network with accurate and precise values of the branch lengths and the extremity load impedances. Significantly, this can be clearly noticed from the agreement of the reconstructed NUT's TDR response and the measured one as seen in Fig. 3.

\section{CONClusion}

In this paper, we have proposed a non-destructive testing approach, the TDR-GA methodology, which is based on the

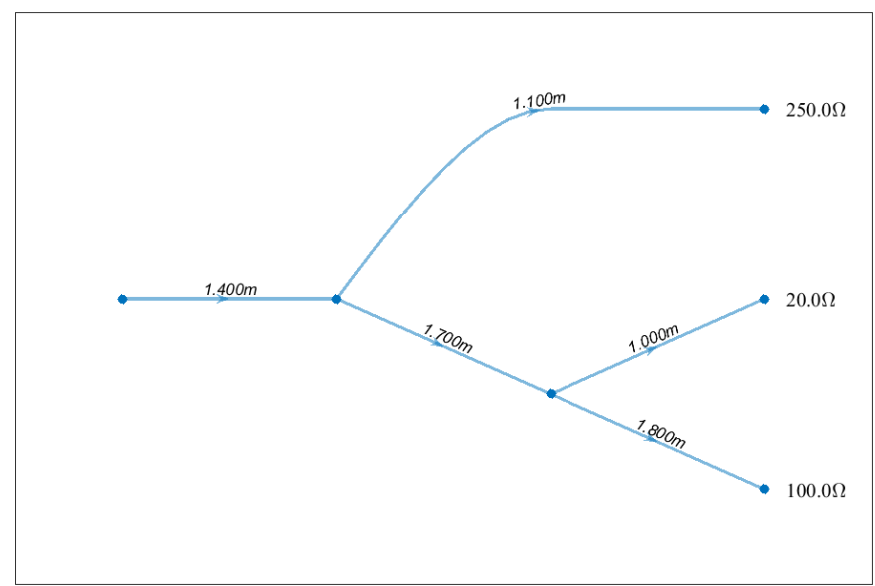

Fig. 5. The constructed NUT after applying the TDR-GA approach on the measured TDR response of the NUT of Fig. 2.

tenets of TDR and genetic algorithms for retrieving the topology of a black-boxed network. The novelty of this method is demonstrated by reconstructing a totally unknown wiring network from a single port TDR measurement while unveiling not only the branch lengths but also the load impedances at the NUT's extremities. The experimental result has proven the effectiveness and feasibility of the proposed approach in precisely reconstructing an unknown double Y-junction complex NUT. The surprising ability of TDR-GA to ensure a $1 \mathrm{~cm}$ length precision along with a $1 \Omega$ impedance accuracy in a relatively short processing time is good news, as no similar performance has been presented so far.

Future work will need to deal with integrating the network connections as an additional variable set in the GA algorithm instead of iterating for each hypothesized NUT.

\section{REFERENCES}

[1] P. Smith, C. Furse, and J. Gunther, "Analysis of Spread Spectrum Time Domain Reflectometry for Wire Fault Location," IEEE Sensors Journal, vol. 5, no. 6, pp. 1469-1478, 2005.

[2] L. A. Griffiths, R. Parakh, C. Furse, and B. Baker, "The Invisible Fray: A Critical Analysis of the Use of Reflectometry for Fray Location," Sensors Journal, IEEE, vol. 6, no. 3, pp. 697-706, 2006.

[3] L. Abboud, A. Cozza, and L. Pichon, "A Matched-Pulse Approach for Soft-Fault detection in complex wire networks," IEEE Trans. Instrumentation and Measurement, vol. 61, no. 6, pp. 1719-1732, 2012.

[4] C. Furse, Y. C. Chung, C. Lo, and P. Pendayala, "A Critical Comparison of reflectometry methods for location of wiring faults," Smart Structures and Systems, vol. 2, no. 1, pp. 25-46, 2006.

[5] L. Abboud, A. Cozza, and L. Pichon, "A Non-Iterative Method for Locating Soft Faults in Complex Wire Networks," IEEE Transactions on Vehicular Technology, vol. 62, no. 3, pp. 1010-1019, 2013.

[6] M. Kafal, A. Cozza, and L. Pichon, "Locating Multiple Soft Faults in Wire Networks using an Alternative DORT Implementation," IEEE Transactions on Instrumentation and Measurement, vol. 65, no. 2, pp. 399-406, 2016.

[7] M. Kafal, A. Cozza, and L. Pichon, "Locating Faults with High Resolution Using Single-Frequency TR-MUSIC Processing," IEEE Transactions on Instrumentation and Measurement, vol. 65, no. 10, pp. 2342-2348, 2016.

[8] F. Auzanneau, "Wire Troubleshooting and Diagnosis: Review and Perspectives," Progr. In Electromag. Res. B, vol. 49, pp. 253-279, 2013.

[9] Z. Michalewicz, "Genetic Algorithms Plus Data Structures Equals Evolution Programs", Springer, 1992. 